\title{
On the regular structure of prefix rewriting
}

\author{
Didier Caucal \\ IRISA, Campus de Beaulieu, F35042 Rennes Cedex, France
}

\begin{abstract}
Caucal, D., On the regular structure of prefix rewriting, Theoretical Computer Science 106 (1992) $61-86$.

We consider a pushdown automaton as a word-rewriting system with labelled rules applied only in a prefix way. The notion of pushdown transition graph is then extended to the notion of prefix transition graph generated by a word-rewriting system and accessible from a given axiom. Such accessible prefix transition graphs are context-free graphs in the sense of Muller and Schupp (1985), and we show that they are also the ronted pattern graphs of finite degree, where a pattern graph is a graph produced from a finite graph by iterating the addition of a finite family of finite graphs (the patterns). Furthermore, this characterization is effective in the following sense: any finite family of patterns generating a rooted graph $G$ of finite degree, is mapped effectively into a word-rewriting system $R$ such that the accessible prefix transition graph of $R$ is isomorphic to $G$, and the reverse transformation is effective.
\end{abstract}

\section{Introduction}

A labelled rewriting system on an alphabet $X$ and a set $L$ of labels, is a finite subset of $X^{*} \times L \times X^{*}$. Every element $(u, f, v)$ of $X^{*} \times L \times X^{*}$ corresponds to a labelled transition $u \stackrel{f}{\longrightarrow} v$. One step of prefix rewriting generated by a rewriting system $R$ is a labelled transition $u w \stackrel{f}{\longrightarrow} v w$, where $u \stackrel{f}{\longrightarrow} v$ is a rule of $R$. Prefix rewriting steps may be viewed as the arcs of a graph, called a prefix transition graph; an accessible prefix transition graph is the graph generated in this way from a given axiom. If for a rule $u \stackrel{f}{\longrightarrow} v, u$ is a letter, we say that $R$ is alphabetic and that the corresponding accessible prefix transition graphs are alphabetic.

As an example of prefix transitions, let us briefly introduce the transitions between the configurations (without input string) of a pushdown automaton (PDA). Such a configuration may be represented as a word $q A_{1} \ldots A_{n}$, where $q$ is a state of the automaton and $A_{1}$ is the top of the stack $A_{1} \ldots A_{n}$. Then the transition relation of the 
PDA can be seen as a rewriting system; a transition between configurations is mapped in this way into a step of prefix rewriting. The corresponding accessible prefix transition graph is called a pushdown transition graph. In Section 1, we show that accessible prefix transition graphs coincide with pushdown transition graphs, but there exist accessible prefix transition graphs that are not alphabetic.

In a seminal paper, Muller and Schupp [14] have proved that every pushdown transition graph has a regular structure: it is a rooted graph of finite degree with a finite number of nonisomorphic connected components obtained after removing all vertices within arbitrary distances of a given vertex. Such a regular structure can be generated by a deterministic graph grammar. In Section 2, we give a procedure which produces a graph grammar generating the transition graph of a given PDA. Conversely, we will also show that any rooted graph of finite degree, generated by a detcrministic graph grammar, is isomorphic to the transition graph of some PDA, and we, moreover, give a procedure which produces the corresponding PDA from the grammar. Then, and in an effective way, we show that the connected components of prefix transition graphs coincide with connected and finite-degree graphs generated by deterministic graph grammars. Furthermore, we establish that every prefix transition graph can effectively be generated by a deterministic graph grammar. Finally, we show how an arbitrary deterministic graph grammar (generating a connected graph of finite degree) can be put into a particular normal form, corresponding to the decomposition of Muller and Schupp, and we effectively obtain in this way their correspondence. As a corollary, we can decide whether two accessible prefix transition graphs (or two connected components of prefix transition graphs) are isomorphic with respect to some given vertices.

\section{Prefix rewriting and pushdown automaton}

In this section, we recall basic facts about rewriting systems, and introduce prefix rewriting as a special case of rewriting, constrained to operate on left factors of words. We then illustrate prefix rewriting with the help of pushdown automata (PDA) and their transitions. The transitions of a PDA are a particular case of prefix rewritings but their transition graphs are shown to be the same.

Let us first introduce notations and terminology for rewriting systems.

Definition 1.1. Given an alphabet $X$ and a set $L$ of labels, a (labelled) rewriting system $R$ is a finite subset of $X^{*} \times L \times X^{*}$.

Every element $(u, f, v)$ of $X^{*} \times L \times X^{*}$ is denoted by $u \stackrel{f}{\longrightarrow} v$. Note that rules $\varepsilon \stackrel{f}{\longrightarrow} v$ are allowed. A rewriting system is said to be alphabetic if $u \in X$ for all rules

$u \stackrel{f}{\longrightarrow} v$, and normal ( $\varepsilon$-free) if both $u$ and $v$ have length smaller than 3 ( $u$ and $v$ are nonempty). 
Rewritings in a rewriting system are generally defined as applications of rewriting rules in every context. On the contrary, we are concerned in this paper with prefix rewriting defined as follows.

Definition 1.2. Given a rewriting system $R$, we define a prefix rewriting step $\stackrel{f}{\mapsto}$ for each label $f$ as follows:

$$
\stackrel{f}{\mapsto}:=\left\{(u w, v w) \mid(u \stackrel{f}{\longrightarrow} v) \in R \wedge w \in X^{*}\right\} .
$$

We represent by $u \mapsto v(u \stackrel{*}{\mapsto} v)$ an elementary (unlabelled) prefix rewriting step (an arbitrary sequence of such steps).

A well known property [5] is that the set $\{w \mid r \stackrel{*}{\mapsto} w\}$ of words in $X^{*}$ reachable by prefix rewriting from a given axiom $r \in X^{*}$ is a regular language, and a corresponding finite automaton is effectively constructible from $R$. Such an automaton is polynomially constructible in time and space [6]. From [4], we can deduce a stronger result.

Theorem 1.3. For any rewriting system $R$, the prefix rewriting $\underset{R}{\stackrel{*}{*}}$ is a rational transduction, and a corresponding transducer is effectively constructible from $R$.

The proof is given in [6]: we establish that the prefix rewriting is the componentwise concatenation of a recognizable relation with the identity relation, so is a right synchronized rational relation $[10,11]$.

Prefix rewriting may be seen as a way to generate labelled transition graphs: the prefix transition graph $P(R)$ is the set of prefix-rewriting steps, i.e.

$$
P(R)=\{u \stackrel{f}{\longrightarrow} v \mid u \stackrel{f}{\longmapsto} v\}=\left\{x w \stackrel{f}{\longrightarrow} y w \mid(x \stackrel{f}{\longrightarrow} y) \in R \wedge w \in X^{*}\right\} .
$$

The prefix transition graph $P(R, r)$ accessible from an axiom $r \in X^{*}$ is the set of prefix-rewriting steps reachable from $r$, i.e.

$$
P(R, r)=\{u \stackrel{f}{\longrightarrow} v \mid u \stackrel{f}{\mapsto} v \wedge r \stackrel{*}{\mapsto} u\} .
$$

Figure 1 gives an example of an accessible prefix transition graph.

In the remainder of the section, we establish a strong connection between prefix rewritings and pushdown automata. To begin with, let us recast pushdown automata and their transitions in the framework of prefix rewriting.

Definition 1.4. A pushdown automaton (without initial and final states) is a rewriting system $R$ satisfying the following conditions:

(i) the alphabet is partitioned into $Q_{R} \cup P_{R}$

(ii) for any rule $u \stackrel{f}{\longrightarrow} v$ in $R$, we have $u \in Q_{R} \cdot P_{R}$ and $v \in Q_{R} \cdot P_{R}^{*}$. 
Let $R$ be the rewriting system on $(\{A, p, q\},\{a, b, c, d\})$ defined as follows:

$$
R=\{p \stackrel{a}{\longrightarrow} q, p \stackrel{b}{\longrightarrow} p A, p A \stackrel{c}{\longrightarrow} p, q A \stackrel{d}{\longrightarrow} q\} .
$$

The accessible prefix transition graph $P(R, p)$ is represented by

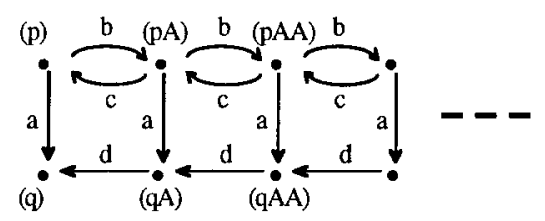

Fig. 1. Accessible prefix transition graph.

This definition corresponds to the usual definition of a pushdown automaton [15] on an input alphabet $\Sigma$ when the label's set is $\Sigma \cup\{\varepsilon\}$. The language recognized by a pushdown automaton $R$ starting at $r \in Q_{R} . P_{R}$ with acceptance on a set $F \subseteq Q_{R}$ of final states, is the set of label sequences $f_{1} \ldots f_{n}$ of the paths $u_{1} \stackrel{f_{1}}{\mapsto} u_{2} \ldots \stackrel{f_{n}}{\mapsto} u_{n+1}$ such that $u_{1}=r$ and $u_{n+1} \in F . P_{R}^{*}$.

Of course, a pushdown automaton (PDA) works under prefix rewriting. Thus, pushdown transition graphs are certainly accessible prefix transition graphs in the following sense.

Definition 1.5. An accessible prefix transition graph (a pushdown transition graph, an alphabetic graph) is a graph isomorphic to $P(R, r)$ for some rewriting system $R$ and some word $r$ (some pushdown automaton $R$ with $r$ in $Q_{R} \cdot P_{R}^{*}$, some alphabetic rewriting system $R$ ).

Here, a graph isomorphism is simply a vertex renaming, but the labels of the arcs are preserved. To establish the converse, i.e. every accessible prefix transition graph is a pushdown transition graph, we show that every accessible prefix transition graph is generated by a normal $\varepsilon$-free transition system.

Lemma 1.6. Any pair $(R, r)$ consisting of a rewriting system $R$ and a word $r$, can effectively be normalized into another pair $(S, s)$, where $S$ is a normal $\varepsilon$-free rewriting system and $s$ is a letter, such that $P(S, s)$ is isomorphic to $P(R, r)$.

Proof. Let $R$ be a rewriting system on $X$, and $r \in X^{*}$. We may suppose $R \varepsilon$-free and $r \neq \varepsilon$. Otherwise, we could take a letter $a$ in $X$ appearing neither in $R$ nor in $r$, and replace $(R, r)$ by $(a R$, ar $)$ with $a R=\{a u \stackrel{f}{\longrightarrow} a v \mid(u \stackrel{f}{\longrightarrow} v) \in R\}$; so, $a R$ is $\varepsilon$-free, $a r \neq \varepsilon$ and $P(a R, a r)=a P(R, r)$. 
Let $m$ be the greatest length of $r$ and the words of $X^{*}$ in $R$, i.e.

$$
m=\max \{|u| \mid(u=r) \vee \exists f \exists v((u \stackrel{f}{\longrightarrow} v) \in R \vee(v \stackrel{f}{\longrightarrow} u) \in R)\} .
$$

Let us extend an injection $i$ from $\left\{u \in X^{+}|1 \leqslant| u \mid \leqslant m\right\}$ to some given alphabet $Y$ to an injection $j$ from $X^{*}$ to $Y^{*}$ by induction

$$
\begin{aligned}
& j(\varepsilon)=\varepsilon, \\
& j(u)=j(v) i(w), \quad \text { where } u=v w \neq \varepsilon \wedge|w|=\min (m,|u|) .
\end{aligned}
$$

The rewriting system $S$ on $Y$ is defined as follows:

$$
S=\left\{j(u w) \stackrel{f}{\longrightarrow} j(v w)\left|(u \stackrel{f}{\longrightarrow} v) \in R \wedge w \in X^{*} \wedge\right| w \mid<m\right\}
$$

is normal and $\varepsilon$-free. Moreover, $s=j(r)$ is a letter. If $R$ is alphabetic, observe that $S$ is alphabetic. We show that

$$
P(S, s)=\{j(u) \stackrel{f}{\longrightarrow} j(v) \mid(u \stackrel{f}{\longrightarrow} v) \in P(R, r)\} .
$$

The proof is an easy but bothering check.

Such a transformation is not usual and corresponds to Lemma 2.4 of [14]. Since accessible prefix transition graphs of normal and $\varepsilon$-frec systems are pushdown transition graphs, we can transform every prefix rewriting system into a PDA without duplication, nor reduction in the accessible prefix transition graph.

Proposition 1.7. Accessible prefix transition graphs coincide effectively with pushdown transition graphs.

Proof. Let $R$ be a rewriting system on $X$ and $r \in X^{*}$. To show that $P(R, r)$ is a pushdown transition graph, we may assume, by Lemma 1.6, that $R$ is a normal and $\varepsilon$-free system, and $r$ is a letter. Consider the following alphabets:

$$
Q=\{u(1) \mid(u=r) \vee \exists f \exists v((u \stackrel{f}{\longrightarrow} v) \in R \vee(v \stackrel{f}{\longrightarrow} u) \in R)\}
$$

of the first letters of $R$ and $r$, and

$$
\Gamma=\{u(i)|2 \leqslant i \leqslant| u \mid \wedge \exists f \exists v((u \stackrel{f}{\longrightarrow} v) \in R \vee(v \stackrel{f}{\longrightarrow} u) \in R)\}
$$

of the $i$ th letters of $R$ with $i>1$. 
Given an injection $i$ from $\Gamma$ to an alphabet $P$ disjoint of $Q$, we extend $i$ to a total injection from $Q \Gamma^{*}$ to $Q P^{*}$ as follows:

$$
i(a u)=a i(u(1)) \ldots i(u(|u|)), \quad \text { with } a \in Q \text { and } u \in \Gamma^{*} .
$$

The rewriting system $S$ on $P \cup Q$ defined by

$$
S=\{i(u) \stackrel{f}{\longrightarrow} i(v) \mid(u \stackrel{f}{\longrightarrow} v) \in R\}
$$

is a normal system. Furthermore, we show that

$$
P(S, s)=\{i(u) \stackrel{f}{\longrightarrow} i(v) \mid(u \stackrel{f}{\longrightarrow} v) \in P(R, r)\},
$$

where $s=i(r) \in Q$.

The system $S$ is not yet a PDA because the domain $\operatorname{Dom}(S)=\{u \mid \exists v, u S v\}$ of $S$, must be included into Q.P. So, we take a new element $p$. The system

$$
T=\{(u \stackrel{f}{\longrightarrow} v) \in S|| u \mid=2\} \cup\left\{u a \stackrel{f}{\longrightarrow} v a|(u \stackrel{f}{\longrightarrow} v) \in S \wedge| u \mid=1 \wedge a \in P_{T}\right\}
$$

is a pushdown automaton with $P_{T}=P \cup\{p\}$ and $Q_{T}=Q$.

Furthermore, $P(T, s p)$ is isomorphic to $P(S, s)$, hence to $P(R, r)$.

After Proposition 1.7, we may ask whether alphabetic rewriting systems are also representatives of arbitrary rewriting systems as far as the generated graphs are concerned. The next proposition gives a negative answer.

Proposition 1.8. The class of alphabetic graphs is a proper subset of the class of accessible prefix transition graphs.

Proof. Any alphabetic graph is an accessible prefix rewriting graph. But let us show that the accessible prefix transition graph of Fig. 1 is not alphabetic. Consider the following system:

$$
R=\left\{p \stackrel{a}{\longrightarrow} q, p \stackrel{b}{\longrightarrow} p A, p A \stackrel{{ }^{c}}{\longrightarrow} p, q A \stackrel{{ }^{d}}{\longrightarrow} q\right\},
$$

and suppose that there exist an alphabet $X$, an alphabetic system $S$ on $(X,\{a, b, c, d\})$ and a word $s$ in $X^{*}$ such that $P(R, p)$ is isomorphic to $P(S, s)$ according to a bijection $f$. Let $i$ be an integer. As $f$ is injective, $f\left(p A^{i}\right) \neq f\left(p A^{j}\right)$ for every $j \neq i$. In particular $\left\{n|| f\left(p A^{n}\right) \mid=i\right\}$ is finite. So, there exists $j$ such that $\left|f\left(p A^{n}\right)\right| \geqslant|f(q)|$ for every $n \geqslant j$. As the set $\left\{\left|f\left(p A^{\prime \prime}\right)\right| \mid n \geqslant j\right\}$ is infinite, there is an integer $m$ such that $\left|f\left(p A^{m+1}\right)\right|>\left|f\left(p A^{m}\right)\right| \geqslant|f(q)|$. Set $u=f\left(p A^{m}\right), v=f\left(p A^{m+1}\right)$ and $w=f\left(q A^{m+1}\right)$.

Because $S$ is alphabetic, $|v|>|u|$ and $(v \stackrel{c}{\longrightarrow} u) \in P(S, s)$; there is $B \in X$ with $v=B u$. The system $S$ being alphabetic and $(v \stackrel{a}{\longrightarrow} w) \in P(S, s)$, there exists $x \in X^{*}$ such that 
$w=x u$. As there exists a unique path in $P(S, s)$ from $w$ to $f(q)$, and $|u| \geqslant|f(q)|$ in the alphabctic system $S$, there is an $n$ such that $0 \leqslant n \leqslant m+1$ and $f\left(q A^{n}\right)=u$. It follows that $f\left(p A^{m}\right)=f\left(q A^{n}\right)$. Then $p A^{m}=q A^{n}$, hence $p=q$, which is a contradiction.

Nevertheless, in the restricted case where $P(R, r)$ has at least one coroot state (reachable from every other state), we have the following result.

Theorem 1.9. From any pair $(R, r)$ consisting of a rewriting system $R$ and a word $r$ such that the accessible prefix transition graph $P(R, r)$ has a coroot, we can decide whether $P(R, r)$ is an alphabetic graph. In this case, the pair $(R, r)$ may be effectively transformed into a pair $(S, s)$, where $S$ is an alphabetic rewriting system and $s$ is a letter, such that $P(S, s)$ is isomorphic to $P(R, r)$.

The construction obtained with Monfort, is given in [7].

\section{Prefix rewriting and pattern graph}

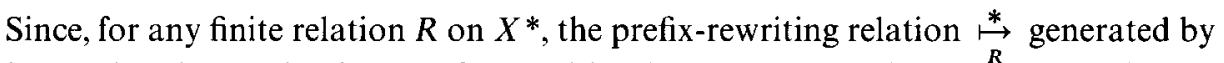
$R$ is a rational transduction, prefix rewriting has a regular behaviour. In particular, the set of vertices of any accessible prefix transition graph is a regular language (over $X^{*}$ ). A natural question is whether the regular structure of accessible prefix transition graph is preserved when transitions are labelled, as in Section 1. The answer is positive, since those graphs are pushdown transition graphs (by Proposition 1.7), and since Muller and Schupp [14] show that pushdown transition graphs coincide with context-free graphs: a context-free graph is a rooted and finite-degree graph which has a finite number of nonisomorphic connected components obtained after removing all vertices within arbitrary distances of a given vertex. Thus, context-free graphs can be cut into slices of a finite number of "patterns".

Building up over the ideas of Muller and Schupp, we devise an effective construction of patterns for accessible prefix transition graphs. We also relax the constraint of splitting up the graph "by slices" and allow the removal of patterns of arbitrary shapes and sizes, to ease the construction of patterns. Furthermore, we establish the converse result: we give a procedure which, given any finite family of patterns (of arbitrary shapes and sizes), produces a PDA whose transition graph is obtained by pasting these patterns together (along a regular tree of formal patterns).

To begin with, let us introduce patterns and their gluing. In order to ease the presentation, we use graph grammars, and first recall their definition (for a good list of references, see [12]).

Definition 2.1. Let $V$ be a set of vertices and $F=\bigcup\left\{F_{n} \mid n \geqslant 1\right\}$ be a graded alphabet. Every word $f v_{1} \ldots v_{n}$ of $F_{n} . V^{n}$ is a hyperarc labelled by $f$ and connecting in order the vertices $v_{1}, \ldots, v_{n}$. A hypergraph is a set of hyperarcs. 
A graph grammar on $(F, V)$ is a finite set of hyperarc replacement rules $f v_{1} \ldots v_{n} \rightarrow H$, where $f v_{1} \ldots v_{n}$ is a hyperarc labelled by the nonterminal $f$, the $v_{i}$ are distinct vertices and $H$ is a finite hypergraph. Every label of a hyperarc of $H$ which is not a nonterminal, is a terminal and is of arity 2.

A graph grammar is deterministic if there is only one rule for each nonterminal $f$. Figure 2 is an example of a deterministic graph grammar (a hyperarc $f v_{1} \ldots v_{n}$ is represented as a label of $v_{1}$ if $n=1$, otherwise is represented as an arc labelled by $f$ from $v_{1}$ to $v_{n}$ with intermediate vertices $v_{2}, \ldots, v_{n-1}$; another representation can be found in [12]).

Let us give some remarks and notations. A hypergraph has no isolated vertex. The first letter $X(1)$ of a hyperarc $X$ is the label of $X$, and $V_{X}=\{X(2), \ldots, X(|X|)\}$ is the set of vertices of $X$; we say that $X$ is a nonterminal hyperarc if $X(1)$ is a nonterminal. There is identity between a hyperarc $X$ and the hypergraph $\{X\}$ reduced to $X$. So, a graph grammar $G$ is a binary relation on the set of (finite) hypergraphs, its domain $\operatorname{Dom}(G)=\{X \mid(X, H) \in G\}$ is the set of left-hand sides of its rules, and its image (or range) $\operatorname{Im}(G)$ is the set of right-hand sides of $G$. We extend by union the set of vertices of a hyperarc to the set $V_{H}$ of vertices of a hypergraph $H$, i.e. $V_{H}=\bigcup\left\{V_{X} \mid X \in H\right\}$.

Each deterministic graph grammar defines a graph, resulting from a given start graph by iterating the graph rewriting $[12,13]$. Intuitively, a rewriting step consists in choosing a nonterminal hyperarc $f t_{1} \ldots t_{n}$ whose label $f$ indicates the rule $f s_{1} \ldots s_{n} \rightarrow H$ to be applied, and the vertices $s_{i}$ in $H$ indicate how to replace $f t_{1} \ldots t_{n}$ by $H$.

Definition 2.2. Given a graph grammar $G$ on $(F, V)$ and a hypergraph $M$ on $(F, V)$, $M$ gives a hypergraph $N$ in one rewriting step, and we denote $M \rightarrow_{G} N$, if there exists a nonterminal hyperarc $f t_{1} \ldots t_{n}$ of $M$ such that

$$
N=\left(M-\left\{f t_{1} \ldots t_{n}\right\}\right) \cup\left\{h g\left(x_{1}\right) \ldots g\left(x_{m}\right) \mid h x_{1} \ldots x_{m} \in H\right\}
$$

Let $A, a, b$ be in $F$ of respective arity $3,2,2$.

Let $G=\{(A 123,\{a 12, a 14, a 25, b 63, A 564\})\}$ be a deterministic graph grammar.

$A$ is the unique nonterminal of $G$, and $G$ is represented as follows:

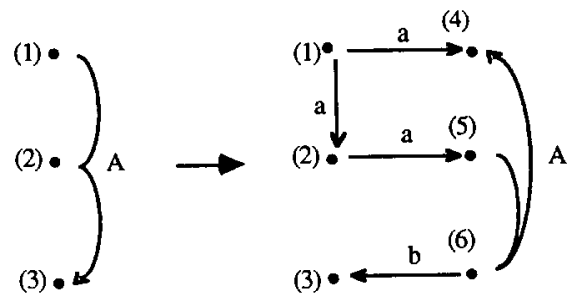

Fig. 2. Deterministic graph grammar. 
for some rule $f s_{1} \ldots s_{n} \rightarrow H$ in $G$ and for some matching function $g$ mapping $s_{i}$ to $t_{i}$, and the other vertices of $H$ injectively to vertices outside of $M$.

Note that $\rightarrow_{G}$ is not, in general, a functional relation, even when $G$ is deterministic. Nevertheless, if we let $M \rightarrow_{G, X} N$ denote the rewriting of a nonterminal hyperarc $X$, then

$$
M \rightarrow_{G, X_{1}} \circ \cdots \circ \rightarrow_{G, X_{n}} N \text { if and only if } M \rightarrow_{G, X_{\pi(1)}} \circ \cdots \rightarrow_{G, X_{\pi(n)}} N
$$

for any $X_{i} \in M$, and for any permutation $\pi$ on $\{1, \ldots, n\}$. Thus, it makes sense to define steps of complete parallel rewriting $M \Rightarrow_{G} N$ as follows:

$$
M \Rightarrow_{G} N \text { if } M \rightarrow_{G, X_{1}} \circ \cdots \circ \rightarrow_{G, X_{n}} N
$$

and $M$ has exactly $n$ nonterminal hyperarcs $X_{1}, \ldots, X_{n}$. One step of complete parallel rewriting corresponds to the Kleene substitution.

Henceforth, the grammar $G$ will be deterministic. The infinite graph $G^{\omega}(M)$ generated by $G$ starting from $M$ is defined below, where $[M]=\{f s t \in M \mid f$ is a terminal $\}$ is the set of terminal arcs of $M$.

Definition 2.3. $G^{\omega}(M)=\bigcup_{n}\left[G^{n}(M)\right]$, where $G^{0}(M)=M$ and $G^{n}(M) \Rightarrow_{G} G^{n+1}(M)$ for all $n$.

Since $G$ is deterministic, $G^{\omega}(M)$ is unique up to hypergraph isomorphism. When $M$ is finite, this element is called the pattern graph generated by $G$ from $M$. Pattern graphs are the equational graphs of Bauderon [2] and Courcelle [8]. The grammar of Fig. 2 generates from $A 123$ the pattern graph of Fig. 3.

Let us recall that a graph $G$ is of finite degree if for every vertex $s$ in $G$, the number of arcs to which $s$ belongs is finite, and is of bounded degree if this number is uniformly bounded. It turns out that every finite-degree pattern graph is a bounded degree graph. A vertex $r$ is a root of a graph $G$ if each vertex of $G$ is reachable from $r$. In particular, every accessible prefix transition graph $P(R, r)$ has a finite degree and root $r$.

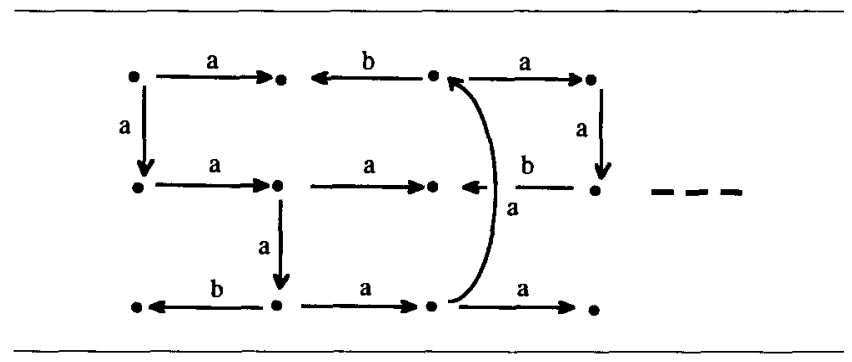

Fig. 3. Pattern graph. 
To characterize prefix transition graphs as pattern graphs, we show that every pattern graph of finite degree can be generated by a normalized graph grammar.

Definition 2.4. A deterministic graph grammar $G$ is in standard form if $G$ satisfies the three following conditions:

(i) $G$ is proper: for all rule $(X, H)$ of $G$, every vertex of $X$ is a vertex of a terminal arc of $H$,

(ii) $G$ is in normal form: for all rule $(X, H)$ of $G$, the vertices of every nonterminal hyperarc of $H$ are disjoint from the $X$ 's ones,

(iii) $G$ is separated: for all rule $(X, H)$ of $G$, two nonterminal hyperares of $H$ have no common vertex, and every nonterminal hyperarc of $H$ has distinct vertices.

The grammar of Fig. 2 is in standard form. Furthermore, we say that $G$ is connected (of finite degree) if for all hyperarc $X$ in $\operatorname{Dom}(G), G^{\omega}(X)$ is connected (of finite degree). Finally, $G$ is reduced according to a nonterminal $f$ if every nonterminal $g$ is "accessible" from $f$, that is the hyperarc $X \in \operatorname{Dom}(G)$ such that $X(1)=f$, rewrites into a hypergraph $H$ (i.e. $X \rightarrow^{*} H$ ) having a hyperarc labelled by $g$ (i.e. there exists $Y \in H$ such that $Y(1)=g)$.

Given a (deterministic) grammar generating a connected and finite-degree graph $H \neq \emptyset$, we can deduce a standard and connected grammar generating $H$.

Lemma 2.5. Any pair $(G, M)$ of a deterministic graph grammar $G$ and a finite hypergraph $M$ such that $G^{\omega}(M)$ is a connected and nonempty graph of finite degree, may be effectively transformed into another pair $(H, N)$, where $H$ is in standard form and connected, $N$ is a hyperarc in $\operatorname{Dom}(H)$, and such that $H^{\omega}(N)$ is equal to $G^{\omega}(M)$.

Proof. (i) We may assume that the set [M] of terminal arcs of $M$ is nonempty: since $G^{\omega}(M)$ is a nonempty graph, it suffices to replace $M$ by a hypergraph $H$ such that $M \Rightarrow{ }^{*} H$ and $[H] \neq \emptyset$. Let $N=S s$, where $S$ is a new symbol in $F_{1}$ and $s$ is a vertex of a terminal arc of $M$. So, $A=G \cup\{(N, M)\}$ is a grammar such that $A^{\omega}(N)=G^{\omega}(M)$.

(ii) We reduce the grammar $A$ according to $h$. Let us consider the accessibility relation

$$
R=\left\{(X(1), Y(1)) \in F^{\prime} \times F^{\prime} \mid \exists H,(X, H) \in A \wedge Y \in H\right\}
$$

on the set $F^{\prime}-\{X(1) \mid X \in \operatorname{Dom}(A)\}$ of the nonterminals of $A$. We construct the set $F^{\prime \prime}=R^{*}(h)$ of the accessible nonterminals from $h=N(1)$, and the restricted grammar

$$
B=\left\{(X, H) \in A \mid X(1) \in F^{\prime \prime}\right\} .
$$

This grammar satisfies $N \in \operatorname{Dom}(B), B^{\omega}(N)=A^{\omega}(N)$ and $B$ is reduced according to $N(1)$.

(iii) Given a graph $H$, we consider the associated symmetric unlabelled graph:

$$
s \leftrightarrow_{H} t \text { if } \exists f,(s \stackrel{f}{\longrightarrow} t) \in H \vee(t \stackrel{f}{\longrightarrow} s) \in H .
$$


For each hyperarc $X$ in $\operatorname{Dom}(B)$, we want to construct the relation

$$
R_{X}=\left\{(s, t) \mid s, t \in V_{X} \wedge s\left(\leftrightarrow_{B^{\omega}(X)}\right)^{+} t\right\}
$$

on the vertices of $X$ which are connected in any pattern graph generated by $B$ from $X$. Let $a$ be a new label of arity 2 , and let us consider the sequence $\left(G_{n}\right)_{n \geqslant 0}$ of graph grammars, defined inductively as follows:

$$
\begin{aligned}
& G_{0}=\{(X, \emptyset) \mid X \in \operatorname{Dom}(B)\} ; \\
& G_{n+1}=\left\{\left(X,\left\{\text { ast } \mid s, t \in V_{X} \wedge s\left(\leftrightarrow_{H}\right)^{+} t\right\}\right) \mid \exists K,(X, K) \in B \wedge K \Rightarrow_{G_{n}} H\right\} .
\end{aligned}
$$

For all $n \geqslant 0, G_{n}$ is a deterministic graph grammar and

$$
G_{n} \subseteq G_{n+1} \subseteq\left\{\left(X,\left\{\text { ast } \mid s, t \in R_{X}\right\}\right) \mid X \in \operatorname{Dom}(B)\right\} .
$$

As the set of vertices $V_{\operatorname{Dom}(B)}$ of $\operatorname{Dom}(B)$ is finite, $h=\min \left\{n \mid G_{n}=G_{n+1}\right\}$ exists and $G_{h}$ can be constructed polynomially in time and space. Furthermore, and for all $X$ in $\operatorname{Dom}(B)$, we have $R_{X}=\leftrightarrow H_{X}$, where $\left(X, H_{X}\right) \in G_{h}$.

(iv) We transform $B$ into a proper grammar preserving $B^{\omega}(N)$. For instance, the following grammar $\{(S 1,\{a 12, P 23\}),(P 12,\{a 13, P 32\})\}$ will be transformed into the grammar $\{(S 1,\{a 12, Q 2\}),(Q 1,\{a 13, Q 3\})\}$ by removing the useless vertex 2 of the hyperarc $P 12$. For every hyperarc $X$ of $\operatorname{Dom}(B)$, a useless vertex of $X$ is a vertex which does not belong to $\operatorname{Dom}\left(R_{X}\right)$. To each $X$ such that $R_{X} \neq \emptyset$, we associate a hyperarc $\underline{X}$ labelled by a new symbol $\underline{X}(1)$ of arity equal to the cardinality \# $\operatorname{Dom}\left(R_{X}\right)$ of $\operatorname{Dom}\left(R_{X}\right)$, whose set $V_{\underline{X}}=\{\underline{X}(2), \ldots, \underline{X}(|\underline{X}|)\}$ of vertices is equal to $\operatorname{Dom}\left(R_{X}\right)$ and such that $\underline{X}(1) \neq \underline{Y}(1)$ if $X \neq Y$. As the vertex $N(2)$ of the axiom $N$ is a vertex of a terminal arc, $N(2) \in \operatorname{Dom}\left(R_{N}\right)$ and we can identify $\underline{N}$ with $N$ (i.e. $\underline{N}(1)$ with $\left.N(1)\right)$. The grammar

$$
I=\left\{(X, \underline{X}) \mid X \in \operatorname{Dom}(B) \wedge R_{X} \neq \emptyset\right\}
$$

removes the useless vertices of hyperarcs in $\operatorname{Dom}(B)$. Then, the grammar

$$
C^{\prime}=\left\{(\underline{X}, K) \mid \exists(X, H) \in B, R_{X} \neq \emptyset \wedge H \Rightarrow_{I} K\right\}
$$

is deterministic and generates $C^{\omega}(N)=B^{\omega}(N)$ because $B^{\omega}(N)$ is connected and $B$ is reduced according to $N(1)$. Let us remark that $R_{X}=R_{\underline{X}}$ for all $X \in \operatorname{Dom}(B)$ such that $R_{X} \neq \emptyset$.

By the construction of $C^{\prime}$, for every hyperarc $X \in \operatorname{Dom}\left(C^{\prime}\right)$, there exists a hypergraph $H_{X}$ such that $X\left(\Rightarrow_{C^{\prime}}\right)^{+} H_{X}$ and each vertex of $X$ is a vertex of a terminal arc of $H_{X}$. The new grammar

$$
C=\left\{\left(X, H_{X}\right) \mid X \in \operatorname{Dom}\left(C^{\prime}\right)\right\}
$$

is proper. Furthermore, $C^{\omega}(N)=C^{\omega}(N)=B^{\omega}(N)$ and $C$ is reduced according to $N(1)$.

(v) We transform $C$ into a proper grammar in normal form. First, we replace the right-hand side $H$ of every rule $(X, H)$ of $C$ by $C^{\omega}(X)$ when the set $V_{C^{\omega}(X)}$ of vertices of $C^{\omega}(X)$ is restricted to $V_{X}$. For instance, the grammar $\{(S 1,\{a 12, A 12\}),(A 12$, $\{a 12, A 21\})\}$ is replaced by the grammar $\{(S 1,\{a 12, A 12\}),(A 12,\{a 12, a 21\})\}$. As $C$ is reduced according to $N(1)$ and $C^{\omega}(N)$ is of finite degree, $C$ is of finite degree. It follows 
that there exists $H_{X}$ such that $X\left(\Rightarrow_{C}\right)^{+} H_{X}$ and every vertex of $X$ is not a vertex of a nonterminal hyperarc of $H_{X}$. Then the grammar

$$
D=\left\{\left(X, H_{X}\right) \mid X \in \operatorname{Dom}(C)\right\}
$$

is in normal form. Furthermore, $D^{\omega}(N)=C^{\omega}(N)$ and the grammar $D$ is proper and reduced according to $N(1)$ because $C$ is. Note that $R_{X}$ is unchanged for every $X$ in $\operatorname{Dom}(D)=\operatorname{Dom}(C)$.

(vi) We transform $D$ into a connected proper grammar in normal form, preserving $D^{\omega}(N)$. After a possible renaming (and adding new rules), we may suppose that every hypergraph of $\operatorname{Im}(D)$ does not have two nonterminal hyperarcs with the same label. For instance, the following proper grammar in normal form $\{(S 1,\{a 12, a 13, P 23\})$, $(P 12,\{a 13, a 24, a 25, P 34, P 35\})\}$ is replaced by the grammar $D_{1}=\{(S 1,\{a 12, a 13, P 23\})$, $(P 12,\{a 13, a 24, a 25, P 34, Q 35\}),(Q 12,\{a 13, a 24, a 25, P 34, Q 35\})\}$. With all $X \in \operatorname{Dom}(D)$ and each class $P$ of $U_{X}=\left\{R_{X}(s) \mid s \in \operatorname{Dom}\left(R_{X}\right)\right\}$, we associate, as in (iv), a hyperarc $X_{P}$ labelled by a new symbol $X_{P}(1)$ of arity \#P, whose set $V_{X_{P}}$ of vertices is cqual to $P$, and such that $X_{P}(1) \neq Y_{Q}(1)$ if $(X, P) \neq(Y, Q)$. As the vertex $N(2)$ of the axiom $N$ is a vertex of a terminal arc of $M, U_{N}=\{\{N(2)\}\}$ and we can identify $N_{\{N(2)\}}(1)$ with $N(1)$.

Consider the grammar

$$
J=\left\{\left(X,\left\{X_{P} \mid P \in U_{X}\right\}\right) \mid X \in \operatorname{Dom}(D)\right\},
$$

which splits each $X \in \operatorname{Dom}(D)$ into hyperarcs according to $U_{X}$. This grammar allows one to split the nonterminal hyperares into a disjoint union of rules in $D$, that is to say for each rule $(X, H)$ of $D$, we associate a hypergraph $H_{X}$ such that $H \Rightarrow_{J} H_{X}$. In fact, each part $X_{P}$ of the splitting of $X$ will generate only the part of $H_{X}$ accessible from $P$. The restriction of a hypergraph $H$ to a set $V$ of vertices is denoted by $H_{\mid V}=\left\{f s_{1} \ldots s_{n} \in H \mid s_{1}, \ldots, s_{n} \in V\right\}$. Then the grammar

$$
E=\left\{\left(X_{P}, H_{X \mid V}\right) \mid X \in \operatorname{Dom}(D) \wedge P \in U_{X} \wedge V=\left(\leftrightarrow H_{X}\right)^{+}(P)\right\}
$$

satisfies the following property

$$
J \circ \Rightarrow_{E}=D \circ \Rightarrow_{J} .
$$

For instance, from grammar $D_{1}$, we obtain the connected grammar $E_{1}=\left\{\left(S 1,\left\{a 12, a 13, P^{\prime} 2, P^{\prime \prime} 3\right\}\right), \quad\left(P^{\prime} 1,\left\{a 13, P^{\prime} 3, Q^{\prime} 3\right\}\right), \quad\left(Q^{\prime} 1,\left\{a 13, P^{\prime} 3, Q^{\prime} 3\right\}\right)\right.$, $\left.\left(P^{\prime \prime} 2,\left\{a 24, a 25, P^{\prime \prime} 4, Q^{\prime \prime} 5\right\}\right),\left(Q^{\prime \prime} 2,\left\{a 24, a 25, P^{\prime \prime} 4, Q^{\prime \prime} 5\right\}\right)\right\}$. Let $T$ be the set of finite hypergraphs $H$ such that two nonterminal (according to $D$ ) hyperarcs of $H$ with the same label, have no common vertex. As every hypergraph of $\operatorname{Im}(D)$ does not have two nonterminal hyperarcs with the same label, $\operatorname{Im}(D)$ is in particular included into $T$. So, we have

$$
\mathrm{id}_{T}^{\circ} \Rightarrow_{J}^{\circ} \Rightarrow_{E}=\mathrm{id}_{T}^{\circ} \Rightarrow_{D}^{\circ} \Rightarrow_{J},
$$

where $\mathrm{id}_{T}=\{(H, H) \mid H \in T\}$ is the identity on $T$. 
As $D$ is a proper grammar in normal form such that $\operatorname{Im}(D) \subseteq T$, the relation $\operatorname{id}_{T} \circ\left(\Rightarrow_{D}\right)^{*}$ is included into $T \times T$. So, by induction on $n \geqslant 0$, we have

$$
\mathrm{id}_{T} \circ \Rightarrow_{I} \circ\left(\Rightarrow_{E}\right)^{n}=\mathrm{id}_{T} \circ\left(\Rightarrow_{D}\right)^{n} \circ \Rightarrow_{I} .
$$

In particular, $E^{\omega}(N)=D^{\omega}(N)$.

Furthermore, $E$ is a proper grammar in normal form, and reduced according to $N(1)$ because $D$ is. As $E^{\omega}(N)=G^{\omega}(M)$ is connected and $E$ is reduced according to $N(1)$, by construction, $E$ is a connected grammar.

(vii) We transform $E$ into a separated grammar. For each rule $(X, H)$ of $E$, we extract the set $W_{X}=\{K \mid \exists H,(X, H) \in E \wedge K$ is a connected component of $H-[H]\}$ of connected components of the nonterminal hyperarcs of $H$, where $X \rightarrow H$ is a rule of $E$.

For all $K \in W_{X}$, we associate a hyperarc $X_{K}$ labelled by a new symbol $X_{K}(1)$ of arity $\# V_{K}, V_{X_{k}}=V_{K}$, and such that $X_{K}(1) \neq X_{K^{\prime}}(1)$ if $K \neq K^{\prime}$. Let us consider the grammar

$$
L^{\prime}=\left\{\left(X,[H] \cup\left\{X_{K} \mid K \in W_{X}\right\}\right) \mid(X, H) \in E\right\}
$$

obtained from $E$ by replacing in all rules $(X, H)$ of $E$, each hypergraph $K$ of $W_{X}$ in $H$ by $X_{K}$. For all $K \in W_{X}$, we associate a hypergraph $H_{X, K}$ such that $K \Rightarrow_{L^{\prime}} H_{X, K}$. The grammar

$$
L=L^{\prime} \cup\left\{\left(X_{K}, H_{X, K}\right) \mid X \in \operatorname{Dom}(E) \wedge K \in W_{X}\right\}
$$

is separated, and satisfies $L^{\omega}(N)=E^{\omega}(N)$. Like grammar $E$, this grammar $L$ is connected, proper and in normal form. Finally, $L$ is a connected grammar in standard form which generates from $N \in \operatorname{Dom}(L)$ the pattern graph $G^{\omega}(M)$.

The next step is to translate a grammar in standard form generating a rooted graph $G$ of finite degree, into a rewriting system generating $G$ by prefix rewritings.

Proposition 2.6. Any triple $(G, M, v)$ of a deterministic graph grammar $G$, a finite hypergraph $M$ and a vertex $v$ of $M$, such that $G^{\omega}(M)$ has finite degree and root $v$, may be effectively transformed into a pair $(R, r)$ of a word-rewriting system $R$ and a word $r$, such that the corresponding graphs $G^{\omega}(M)$ and $P(R, r)$ are isomorphic.

Proof. From Lemma 2.5, we can assume that $G$ is a connected grammar in standard form, and $M$ is a hyperarc. After a possible renaming of labels (and provided, we add new rules), we further suppose that every hypergraph of $\operatorname{Im}(G)$ does not have two nonterminal hyperarcs with the same label.

Let $N$ be the set of nonterminals of $G$, and $V$ be the set of vertices of $G$. For convenience, a vertex of a nonterminal hyperarc is called an output. With each rule $(X, H)$ of $G$, we associate a total function $p_{X}$ from $V_{H}$ to $V \cup V . N$, which is the identity on the set of nonoutput vertices of $H$. For any output vertex $s$ of $H$, we have $p_{X}(s)=T(i) T(1)$, where $T$ is the nonterminal hyperarc in the domain of $G$ with the 
same label as the nonterminal hyperarc $Y$ whose vertex is $s$, and $i$ is the place of $s$ in $Y$, i.e.

$$
\begin{array}{ll}
p_{X}(s)=s & \text { for } s \in V_{H} \text { such that } s \notin V_{J} \text { for all } J \in H \text { such that } J(1) \in N, \\
p_{X}(s)=T(i) T(1) & \text { if there exist } Y \in H \text { and } T \in \operatorname{Dom}(G) \text { such that } Y(i)=s \text { and } \\
& T(1)=Y(1) .
\end{array}
$$

Since $G$ is separated, $p_{X}$ is well-defined.

Let $R(G)$ be the rewriting system on $N \cup V$ and labelled in $F$, defined by

$$
R(G)=\left\{p_{X}(s) . X(1) \stackrel{a}{\longrightarrow} p_{X}(t) . X(1) \mid \exists H,(X, H) \in G \wedge(s \stackrel{a}{\longrightarrow} t) \in H \wedge a \notin N\right\} .
$$

The vertices $w$ of $P(R(G), v \cdot M(1))$ are words $w_{0} n_{p} \ldots n_{1}$, where $w_{0}$ is the vertex of the "pattern" $P$ which first introduced $w$ as nonoutput vertex, and $n_{p} \ldots n_{1}$ is the label sequence of the nonterminal hyperarcs whose rewritings have given $P$. Then $P(R(G), v . M(1))$ is isomorphic to $G^{\omega}(M)$.

Applied to the grammar of Fig. 2, the construction of Proposition 2.6 gives the following rewriting system

$$
R=\{1 A \stackrel{a}{\longrightarrow} 2 A, 1 A \stackrel{a}{\longrightarrow} 3 A A, 2 A \stackrel{a}{\longrightarrow} 1 A A, 2 A A \stackrel{b}{\longrightarrow} 3 A\} .
$$

Hence, $P(R, 1 A)$ is the pattern graph of Fig. 3 .

The converse of Proposition 2.6 is true: in an effective way, every accessible prefix transition graph is a rooted pattern graph of finite degree.

Proposition 2.7. Any pair $(R, r)$ of a word-rewriting system $R$ and a word $r$, may be effectively transformed into a pair $(G, M)$ of a deterministic graph grammar $G$ and a hyperarc $M$, such that the corresponding graphs $P(R, r)$ and $G^{\omega}(M)$ are isomorphic.

Proof. From Lemma 1.6, we may suppose that $R$ is normal and $\varepsilon$-free on $X$, and $r \in X$. The grammar $G$ to be constructed generates $P(R, r)$ by vertices of increasing length.

We consider the connected component $P(R, r)_{\mid u}$ of $P(R, r)$ restricted to the vertices of length at least $|u|$, and containing $u$.

We can determine the set $V(u)$ of vertices $P(R, r)_{\mid u}$ of length $|u|$. From [5] or [6], we can construct an automaton recognizing the set $\underset{R}{\stackrel{*}{R}}(r)=\{v \mid r \underset{R}{\stackrel{*}{*}} v\}$ of vertices of $P(R, r)$. So, we can determine the finite set $D(u)=\underset{R}{\stackrel{*}{*}}(r) \cap X^{|u|}$ of vertices of $P(R, r)$ of the same length as $u$. To decide if two elements in $D(u)$ are connected in the restriction of $P(R, r)$ to the vertices having length $\geqslant|u|$, we construct the unlabelled rewriting system $S$, defined by

$$
S=\{(x z, y z)|| z \mid=\max (0,|u|-|x|) \wedge \exists f(x \stackrel{f}{\longrightarrow} y) \in R\},
$$


and we determine the following relation $E$ on $D(u)$ :

$$
E=\{(x, y) \mid x, y \in D(u) \wedge \underset{s}{\stackrel{*}{*}}(x) \cap \underset{s}{\stackrel{*}{\rightarrow}}(y))-\{z|| z|<| u \mid\} \neq \emptyset\} .
$$

So, $V(u)$ is the class in the partition of $D(u)$ by the equivalence $E^{*}$, containing $u$, i.e.

$$
V(u)=E^{*}(u)=\left\{v \mid u E^{*} v\right\} .
$$

Furthermore, consider the symmetric system $T$ of unlabelled word rewritings on $X^{|u|} X^{*}$, defined by

$$
\begin{array}{r}
T=\{(x z, y z)|| z \mid=\max (0,|u|-|x|,|u|-|y|) \\
\wedge \exists f((x \stackrel{f}{\longrightarrow} y) \in R \vee(y \stackrel{f}{\longrightarrow} x) \in R)\} .
\end{array}
$$

So, the set of vertices of $P(R, r)_{\mid u}$ is included in $\{v \mid u \underset{T}{\stackrel{*}{*}} v\}$, i.e. $V_{P(R, r)_{\mid u}} \subseteq \underset{T}{\stackrel{*}{*}}(u)$.

As $R$ is normal, the set of vertices of $P(R, r)_{\mid u}$ have a common suffix $s_{u}$ of length $\max (0,|u|-2)$. We denote by $V \cdot u^{-1}=\{v \mid v u \in V\}$ the right quotient of a language $V$ by a word $u$.

Two vertices $u$ and $v$ of $P(R, r)$ are equivalent, denoted as $u \equiv v$, if $V(u) . s_{u}^{-1}=V(v) . s_{v}^{-1}$. If $u \equiv v$ then $P(R, r)_{\mid u}$ is isomorphic to $P(R, r)_{\mid v}$. Moreover, the equivalence $\equiv$ is of finite index and a set $U$ of representatives is constructible from $(R, r)$ with $r \in U$. For any $u \in U$, we associate the graph $H_{u}$ of arcs of $P(R, r)_{\mid u}$ with a vertex of length $|u|$. To construct the grammar $G$, we only add to each $H_{u}$ a set $K_{u}$ of nonterminal hyperarcs which generates, according to $G$, the graph $P(R, r)_{\mid u}$ restricted to vertices of length strictly greater than $|u|$.

To this end, we take a graded alphabet $F$ disjoint from the label set of $R$, and an injection $j$ from $U$ to the set of hyperarcs labelled by $F$ with vertices in $X^{*}$, such that for every $u$ in $U$, we have

$$
j(u)=f s_{1} \ldots s_{n} \text { with }\left\{s_{1}, \ldots, s_{n}\right\}=V(u), s_{i} \neq s_{j} \text { if } i \neq j, f \neq j(v)(1) \text { if } v \in U-\{u\} .
$$

For any $u \in U$, we define

$$
\begin{aligned}
K_{u}= & \left\{g\left(t_{1} s_{w}\right) \ldots\left(t_{n} s_{w}\right)\left|w \in V_{P(R, r)_{u}} \wedge\right| w|=| u \mid+1 \wedge \exists v \in U, v \equiv w\right. \\
& \left.\wedge j(v)=g\left(t_{1} s_{v}\right) \ldots\left(t_{n} s_{v}\right)\right\},
\end{aligned}
$$

and we define the following deterministic graph grammar:

$$
G=\left\{\left(j(u), H_{u} \cup K_{u}\right) \mid u \in U\right\} .
$$

Note that $G$ is a connected and finitc-degrec grammar in standard form.

For any $u \in U, P(R, r)_{\mid u}$ is isomorphic to $G^{\omega}(j(u))$ and, in particular, for $M=j(r)$ $P(R, r)$ is isomorphic to $G^{\infty}(M)$.

The construction of Proposition 2.7 is illustrated in Fig. 4. 
Applied to the pair $(R, p)$ of Fig. 1, the construction of Proposition 2.7 gives the following grammar $G$ :

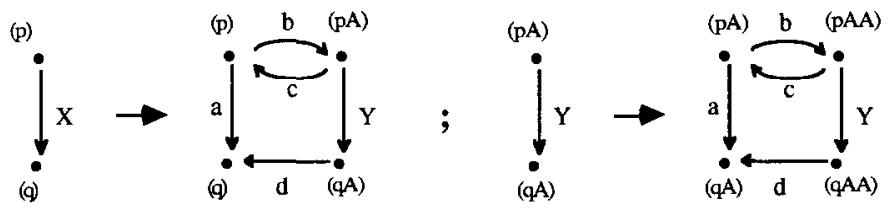

The accessible prefix transition graph $P(R, p)$ is isomorphic to the pattern graph $G^{\omega}(X p q)$.

Fig. 4. Extraction of a graph grammar from an accessible prefix transition graph.

A restricted version of Proposition 2.7 was established in [1] for alphabetic graphs with a coroot of out-degree zero. Propositions 2.6 and 2.7 establish constructively the following statement.

Theorem 2.8. Accessible prefix transition graphs coincide effectively with rooted pattern graphs of finite degree.

After Propositions 2.6 and 2.7, we can determine a word-rewriting system for the inverse of any accessible prefix transition graph with a coroot.

Proposition 2.9. Any triple $(R, r, c)$ consisting of a rewriting system $R$, a word $r$ and a coroot $c$ of $P(R, r)$, may be effectively transformed into another triple $(S, s, d)$ such that there exists an isomorphism from $P(S, s)$ to the inverse of $P(R, r)$ satisfying $f(s)=c$ and $f(d)=r$.

Proof. After renaming, we can suppose that any two rules in $R$ have not the same label, so the coroot $c$ is unambiguously determined by a path from $r$ to $c$. From Proposition 2.7, we can transform $(R, r)$ into $(G, M)$, where $G$ is a deterministic graph grammar, and $M$ is a finite hypergraph such that $P(R, r)$ is a pattern graph $G^{\omega}(M)$ generated by $G$ from $M$. Provided we rewrite $M$ a suitable number of times, we can assume that it contains $r$ and $c$. Let $h$ be a new label of arity 2 and let $G^{\prime}=G \cup\{(h r c, M)\}$. We construct a grammar $H$ by inverting the arcs of the right members of the rules in $G^{\prime}$, i.e.

$$
H=\left\{\left(X, K^{-1}\right) \mid(X, K) \in G^{\prime}\right\} .
$$

So, $H^{\omega}(h r c)$ is the inverse graph of $G^{\omega}(M)$, where $c$ is a root and $r$ is a coroot. Consequently, by restituting the old labels, the system $S$ constructed in Proposition 2.6 satisfies the requirements with $s=c h$ and $d=r h$.

Propositions 2.6 and 2.7 allow the study of other effective transformations of prefix-rewriting systems, not only the computation of the inverse as in Proposition 2.9. One of these transformations allows an effective extension of Theorem 2.8 to the 
connected components of prefix transition graphs. The connected component of a prefix transition graph $P(R)$ containing a vertex $r$, is denoted by $P(R)_{r}$, i.e.

$$
P(R)_{r}=\left\{u \stackrel{f}{\longrightarrow} v \mid r\left(\leftrightarrow_{P(R)}\right)^{*} u \wedge u \stackrel{f}{\mapsto} v\right\}
$$

Such a class of graphs is extended up to isomorphism.

Definition 2.10. A prefix transition graph (a connected prefix transition graph, a rooted prefix transition graph) is a graph isomorphic to $P(R)\left(P(R)_{r}, P(R)_{r}\right.$, having a root) for some rewriting system $R$ (and some word $r$ ).

Figure 5 gives an example of a connected prefix transition graph. Propositions 2.6 and 2.7 help to prove the following statement.

Theorem 2.11. Connected prefix transition graphs coincide effectively with connected pattern graphs of finite degree.

Proof. (i) Let us consider a word-rewriting system $R$ on $X$ labelled in $L$, and a word $r \in X^{*}$. With each symbol $f$ of $L$, we associate injectively a symbol $f^{\prime}$ in a new set $L^{\prime}$, and we define a word-rewriting system $S$ on $X$ labelled in $L \cup L^{\prime}$, as follows:

$$
S=R \cup\left\{v \stackrel{f^{\prime}}{\longrightarrow} u \mid(u \stackrel{f}{\longrightarrow} v) \in R\right\} .
$$

Then $P(R)_{r}$ is the graph obtained from $P(S, r)$ by reversing the arcs labelled in $L^{\prime}$, i.e.

$$
P(R)_{r}=\{(u \stackrel{f}{\longrightarrow} v) \in P(S, r) \mid f \in L\} \cup\left\{u \stackrel{f}{\longrightarrow} v \mid\left(v \stackrel{f^{\prime}}{\longrightarrow} u\right) \in P(S, r) \wedge f^{\prime} \in L^{\prime}\right\} .
$$

Let $R$ be the rewriting system on $(\{A, B, C\},\{a, b, c, d\})$ defined as follows:

$$
R=\left\{A \stackrel{{ }^{a}}{\longrightarrow} A A, A \stackrel{b}{\longrightarrow} B, C \stackrel{{ }^{c}}{\longrightarrow} B, C \stackrel{d}{\longrightarrow} C A\right\} .
$$

The connected component $P(R)_{A}$ of $P(R)$ containing $A$, is represented by

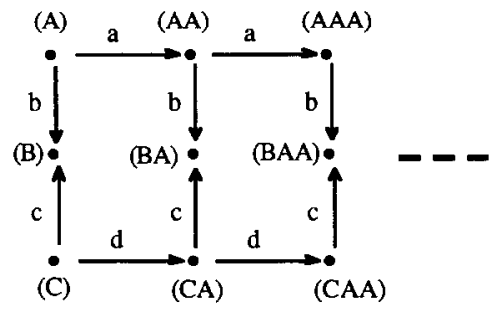

Fig. 5. Connected prefix transition graph. 
From Proposition 2.7, the pair $(S, r)$ may be effectively transformed into a deterministic graph grammar $G$ and a hyperarc $M$ in $\operatorname{Dom}(G)$ such that $P(S, r)$ is a pattern graph generated by $G$ from $M$. In the patterns of $G$, we reverse the terminal arcs whose labels are in $L^{\prime}$, that is to say, we consider the following deterministic graph grammar $H$ :

$$
H=\left\{\left(X,\left\{Y \subset K \mid Y(1) \notin L^{\prime}\right\} \cup\left\{u \stackrel{f}{\longrightarrow} v \mid\left(v \stackrel{f^{\prime}}{\longrightarrow} u\right) \in K \wedge f^{\prime} \in L^{\prime}\right\}\right) \mid(X, K) \in G\right\} .
$$

Then $P(R)_{r}$ is isomorphic to $H^{\omega}(M)$.

(ii) Let us consider a deterministic graph grammar $G$ and a finite hypergraph $M$ such that $G^{\omega}(M)$ is connected and of finite degree. The empty graph is a connected prefix transition graph; otherwise, we can assume that $G^{\omega}(M)$ is not the empty graph. From Lemma 2.5, we can assume that $G$ is a connected grammar in standard form and $M$ is a hyperarc in $\operatorname{Dom}(G)$. Then, the rewriting system $R(G)$ in the proof of Proposition 2.6 is suitable because $P(R(G))_{v . M(1)}$ is isomorphic to $G^{\omega}(M)$ for any vertex $v$ of $M$. So, every connccted pattcrn graph of finite degree is effectively a connected prefix transition graph.

As a consequence, the rooted components of prefix transition graphs are the accessible prefix transition graphs.

Corollary 2.12. Rooted prefix transition graphs coincide effectively with accessible prefix transition graphs.

Proof. (i) Let $P(R)_{u}$ be a connected prefix transition graph with a root $r$. Then $P(R)_{u}=P(R, r)$ is an accessible prefix transition graph.

(ii) Let $P(R, r)$ be an accessible prefix transition graph. Note that the root $r$ of $P(R, r)$ is not necessarily a root of $P(R)_{r}$, i.e. we can have $P(K, r) \neq P(R)_{r}$. By Theorem $2.8, P(R, r)$ is effectively a rooted pattern graph of finite degree. By Theorem 2.11, $P(R, r)$ is effectively a connected prefix transition graph.

Finally, we consider prefix transition graphs. Every finite graph is a pattern graph of finite degree which is not a prefix transition graph because (nonempty) prefix transition graphs are infinite. Nevertheless, and from Theorem 2.11, the converse is true.

Theorem 2.13. Prefix transition graphs are effectively pattern graphs of finite degree.

Proof. We first give a construction, and in the following, steps prove the assertion of the theorem.

(i) Let $R$ be a word-rewriting system on $X$ with labels in $L$. Let $W$ be the set of words in $R$, i.e.

$$
W=\{u \mid \exists v, \exists f,(u \stackrel{f}{\longrightarrow} v) \in R \vee(v \stackrel{f}{\longrightarrow}, u) \in R\},
$$

and we denote by $m=\max \{|u| \mid u \in W\}$ the maximum length of the words in $R$. 
Recall that the symmetric closure of the (unlabelled) prefix-rewriting step is denoted by ' , i.e.

$$
\leftrightarrow=\{(u, v) \mid u \mapsto v \vee v \mapsto u\},
$$

and the equivalence $(\leftrightarrow)^{*}$ is preserved by right concatenation. We have $V_{P(R)}=\operatorname{Dom}(\leftrightarrow)=W . X^{*}$. We consider the set $E=\{u w|u \in W \wedge| u w \mid \leqslant m\}$ of vertices of $P(R)$ having length at most $m$, and we construct $E /(\leftrightarrow)^{*}=\left\{C_{1}, \ldots, C_{p}\right\}$. We choose an element $r_{i}$ of $C_{i}$ of minimal length. From Theorem 2.11 and Lemma 2.5, we can construct a grammar $G_{i}$ in standard form (and connected) and a hyperarc $N_{i}$ in $\operatorname{Dom}\left(G_{i}\right)$ such that $P(R)_{r_{i}}$ is isomorphic to $G_{i}^{\omega}\left(N_{i}\right)$. After a possible renaming, we may assume that the $G_{i}$ have distinct nonterminals and vertices. Take two new unary symbols $A$ and $B$, and a new vertex $v$. We construct the following "repetitive" grammar $H$ :

$$
H=\left\{\left(B v,\{B v\} \cup\left\{N_{i}|1 \leqslant i \leqslant p \wedge| r_{i} \mid=m\right\}\right)\right\}
$$

and the grammar $G$ as follows:

$$
G=\left\{\left(A v,\{B v\} \cup\left\{N_{i} \mid 1 \leqslant i \leqslant p\right\}\right)\right\} \cup H \cup \bigcup\left\{G_{i} \mid 1 \leqslant i \leqslant p\right\} .
$$

To prove this theorem, it is enough to show that $P(R)$ is isomorphic to $G^{\omega}(A v)$.

With each $1 \leqslant i \leqslant p$, we associate the following family

$$
V_{i}=\left\{C \in V_{P(R)} /(\leftrightarrow)^{*} \mid \exists c \in \operatorname{Min}(C), p_{c}=r_{i}\right\},
$$

where Min $(C)$ is the set of words in $C$ of minimal length and $p_{c}$ is the prefix of the word $c$ of length $\min (m,|c|)$.

To prove that $P(R)$ is generated by $G$ from $A v$, it suffices to prove successively:

(1) $\bigcup\left\{V_{i} \mid 1 \leqslant i \leqslant p\right\}$ is a partition of $V_{P(R)}$,

(2) $P(R)_{d}$ is isomorphic to $P(R)_{r_{i}}$ for any $d \in C \in V_{i}$,

(3) if $\left|r_{i}\right|<m$ then $\# V_{i}=1$ else \# $V_{i}=\infty$.

(ii) We shall need the following three claims.

Claim $1:\left|r_{i}\right|=m \wedge u \leftrightarrow^{*} r_{i} v \Rightarrow \exists w, w \leftrightarrow^{*} r_{i} \wedge u=w v$.

As $\left|r_{i}\right|=m$ and by minimality of $\left|r_{i}\right|,|c| \geqslant m$ for every $c$ in $\left[r_{i}\right]=\left\{u \mid r_{i} \leftrightarrow^{*} u\right\}$. By definition of $m$, if $r_{i} v \leftrightarrow^{*} u$ then there exists $w$ such that $r_{i} \leftrightarrow^{*} w$ and $w v=u$.

Claim 2: $u \leftrightarrow^{*} r_{i} \wedge \exists v, u v \in \operatorname{Min}([u v]) \Rightarrow|u|=\left|r_{i}\right|$.

As $u \leftrightarrow^{*} r_{i}$ and by minimality of $\left|r_{i}\right|$, we have $\left|r_{i}\right| \leqslant|u|$. As $\leftrightarrow^{*}$ is closed by right concatenation, $u v \leftrightarrow^{*} r_{i} v$. So, $|u v| \leqslant\left|r_{i} v\right|$; hence, $|u| \leqslant\left|r_{i}\right|$. In consequence, $|u|=\left|r_{i}\right|$. Claim 3: $C \in V_{i} \Leftrightarrow \exists c \in \operatorname{Min}(C), p_{c} \leftrightarrow^{*} r_{i}$.

The necessary condition follows immediately from the definition of $V_{i}$. Conversely, let $c \in \operatorname{Min}(C)$ such that $p_{c} \leftrightarrow^{*} r_{i}$. Let $c^{\prime}$ be the suffix of $c$ such that $c=p_{c} \cdot c^{\prime}$. As $\leftrightarrow^{*}$ is preserved by right concatenation, $c \leftrightarrow^{*} r_{i} \cdot c^{\prime}$. By Claim 2, $\left|p_{c}\right|=\left|r_{i}\right|$; hence, $r_{i} . c^{\prime} \in \operatorname{Min}(C)$. Furthermore, $c^{\prime}=\varepsilon$ if $\left|r_{i}\right|<m$; so, $p_{r_{i} \cdot c^{\prime}}=r_{i}$. Finally, $C \in V_{i}$.

(iii) Proof of (1). Let $C$ be an equivalence class of $V_{P(R)}$ according to $\leftrightarrow^{*}$, and we want to show that there exists a unique $i$ such that $C \in V_{i}$.

Existence: Let $c \in \operatorname{Min}(C)$. As $c \in V_{P(R)}, p_{c} \in V_{P(R)}$. Furthermore, $\left|p_{c}\right| \leqslant m$; hence $p_{c} \in E$. So, there exists (a unique) $1 \leqslant i \leqslant p$ such that $p_{c} \leftrightarrow^{*} r_{i}$; and, by Claim $3, C \in V_{i}$. 
Unicity: Let $c, d \in \operatorname{Min}(C)$ such that $p_{c}=r_{i}$ and $p_{d}=r_{j}$. We have $c \leftrightarrow^{*} d$ and $|c|=|d|$.

If $|c| \leqslant m$ then $p_{c}=c$ and $p_{d}=d$; then $p_{c} \leftrightarrow^{*} p_{d}$ and, hence, $r_{i} \leftrightarrow^{*} r_{j}$.

If $|c|>m$ then $\left|r_{i}\right|=\left|p_{c}\right|=m=\left|r_{j}\right|=\left|p_{d}\right|$. Let $c^{\prime}$ be such that $c=p_{c} \cdot c^{\prime}$. So, $r_{i} \cdot c^{\prime} \leftrightarrow * d$ and, by Claim 1, there exists $w$ such that $w \leftrightarrow^{*} r_{i}$ and $d=w \cdot c^{\prime}$. By Claim 2, $|w|=m$; then $w=p_{d}$ and, so, $r_{i} \leftrightarrow{ }^{*} r_{j}$.

In all cases $r_{i} \leftrightarrow^{*} r_{j}$; hence $i=j$.

(iv) Proof of (2). Let $C \in V_{i}$ : there exists $c \in \operatorname{Min}(C)$ such that $p_{c}=r_{i}$.

If $|c| \leqslant m$ then $p_{c}=c$. So, $c=r_{i}$; then $P(R)_{c}=P(R)_{r_{i}}$.

If $|c|>m$ then $\left|r_{i}\right|=\left|p_{c}\right|=m$. Let $c^{\prime}$ be such that $c=p_{c} . c^{\prime}$. By Claim 1, we obtain

$$
P(R)_{c}=\left\{u \cdot c^{\prime} \stackrel{f}{\longrightarrow} v \cdot c^{\prime} \mid(u \stackrel{s}{\longrightarrow} v) \in P(R)_{r_{i}}\right\}
$$

which is isomorphic to $P(R)_{r_{i}}$.

(v) Proof of (3). Suppose that $\# V_{i} \neq 1$ and we show that $\left|r_{i}\right|=m$. As $\left[r_{i}\right] \in V_{i}$, there exists $C \in V_{i}$ such that $C \neq\left[r_{i}\right]$. So, there exists $c \in \operatorname{Min}(C)$ such that $p_{i}=r_{i}$. Since $C \neq\left[r_{i}\right], p_{c} \neq c$; hence, $|c|>m$. So, $\left|r_{i}\right|=m$. Therefore, if $\left|r_{i}\right|<m$ then $\# V_{i}=1$.

Suppose that $\left|r_{i}\right|=m$ and we show that $\# V_{i}=\infty$. Let $v \in X^{*}$ and $c \in \operatorname{Min}\left(\left[r_{i} v\right]\right)$. As $c \leftrightarrow^{*} r_{i} v,\left|r_{i}\right|=m$ and, by Claim 1 , there exists $w$ such that $w \leftrightarrow^{*} r_{i}$ and $c=w v$. By Claim 2, $|w|=\left|r_{i}\right|=m$. Therefore, $w=p_{c}$ and, hence, $p_{c} \leftrightarrow^{*} r_{i}$. By Claim 3, $\left[r_{i} v\right] \in V_{i}$ for any $v \in X^{*}$. Furthermore, if $\left[r_{i} u\right]=\left[r_{i} v\right]$ then $r_{i} u \leftrightarrow^{*} r_{i} v$ and, by Claim 1, we obtain $u=v$. Eventually $\# V_{i}=\infty$ if $\left|r_{i}\right|=m$.

The construction of Theorem 2.13 is illustrated in Fig. 6 .

From Theorem 2.13 and [8], every closed monadic second-order formula of prefix-rewriting step is decidable.

Corollary 2.14. The monadic second-order theory of the prefix-rewriting step on words is decidable.

For instance, the termination and the confluence of the prefix-rewriting step is decidable (short proofs can be found in [5]).

We shall now reconsider the result of Muller and Schupp [14]. The next definition translates their notion of finite decomposition into the framework of generating grammars.

Definition 2.15. A uniform grammar is a connected graph grammar in standard form in which all rule $f s_{1} \ldots s_{n} \rightarrow H$ of $G$ satisfies the additional conditions:

(i) every terminal arc of $H$ goes through at least one $s_{i}$,

(ii) every vertex of a nonterminal hyperarc of $H$ also belongs to a terminal arc of $H$.

For instance, the grammar of Fig. 2 is uniform. It is obvious to see that context-free graphs, defined in [14], are the rooted pattern graphs generated by uniform grammars. So, a context-free graph is a finite-degree pattern graph. Our goal is to prove constructively Theorem 2.6 of [14]. 
Let $R$ be the rewriting system on $(\{A, B\},\{a, b\})$ defined as follows:

$$
R=\{A A \stackrel{a}{\longrightarrow} A, B \stackrel{b}{\longrightarrow} A B\}
$$

The construction of Theorem 2.13 gives the set $E / \stackrel{*}{\leftrightarrow}=\{\{A, A A\},\{B, A B\},\{B A\},\{B B\}\}$ and the grammar
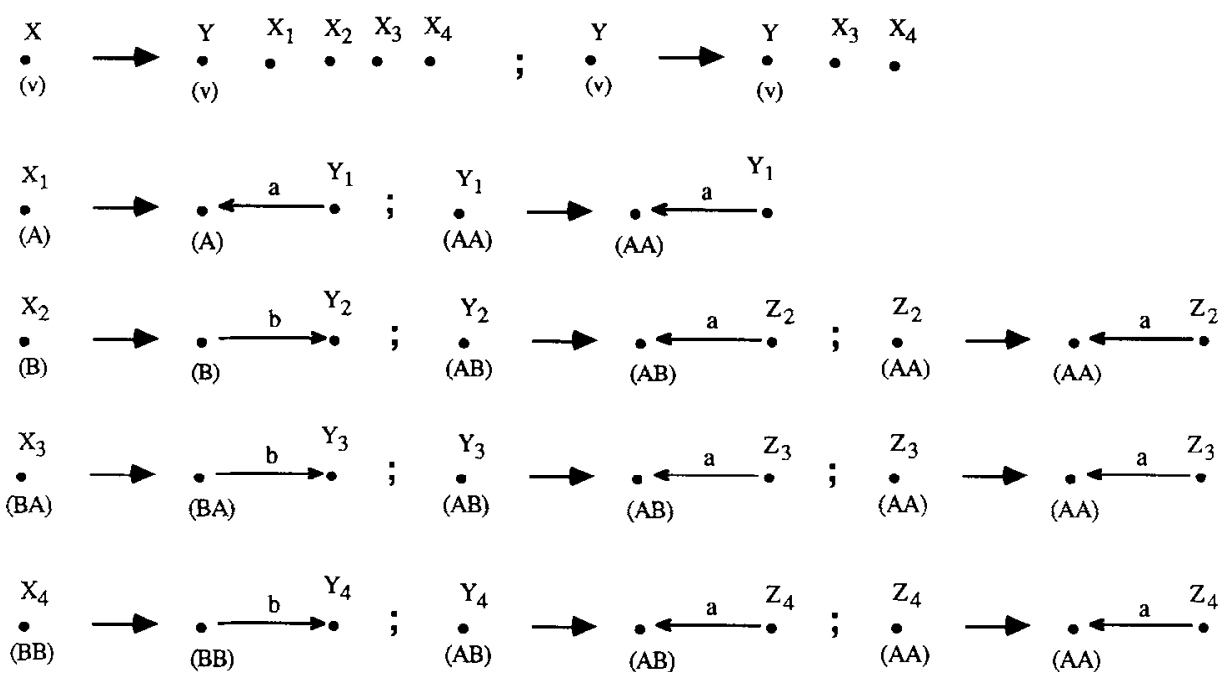

This grammar generates from $X$ the prefix transition graph $P(R)$ of $R$, represented by

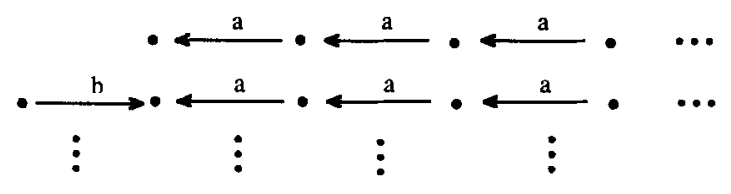

Fig. 6. Extraction of a graph grammar from a prefix transition graph.

Theorem 2.16. Context-free graphs coincide effectively with pushdown transition graphs.

From Propositions 2.6 and 1.7, to every uniform grammar corresponds effectively a pushdown automaton whose transition graph is generated by the grammar. The converse follows from Proposition 2.7 and from the proposition below, which states effectively that every finite-degree and connected pattern graph can be generated by a uniform grammar.

Proposition 2.17. Any pair $(G, M)$ of a deterministic graph grammar $G$ and of a finite hypergraph $M$ such that $G^{\omega}(M)$ is a connected and nonempty graph of finite degree, may be effectively transformed into a uniform grammar $H$ such that $H^{\omega}(M)$ is equal to $G^{\omega}(M)$. 
Proof. From Lemma 2.5, we may assume that $G$ is in standard form and connected, and that $M$ is a hyperarc in $\operatorname{Dom}(G)$. $\Lambda$ fter a possible renaming of labcls (and provided, we add new rules), we further suppose that every hypergraph of $\operatorname{Im}(G)$ does not have two nonterminal hyperarcs with the same label. Finally, and after a possible renaming of vertices, we may assume that hypergraphs of $\operatorname{Im}(G)$ have no common vertex.

Let $V$ be the vertex set of the right members of $G$ and $N$ be the set of nonterminals. We consider a total order $\leqslant$ on $V . N^{*}$ preserved by right concatenation, and for all graph $C$ with vertices in $V . N^{*}$ and every nonterminal word $u$, we write

$$
C . u=\left\{f\left(s_{1} u\right) \ldots\left(s_{n} u\right) \mid f s_{1} \ldots s_{n} \in C\right\}
$$

the suffixing of the vertices of $C$ by $u$,

$$
C . u^{-1}=\left\{f s_{1} \ldots s_{n} \mid f\left(s_{1} u\right) \ldots\left(s_{n} u\right) \in C\right\}
$$

the right quotient of $C$ by $u$, and $s_{C}$ the greater common suffix in $N^{*}$ of vertices in $C$.

We need a representative $R(G, M)$ of the pattern graphs $G^{\omega}(M)$ where vertices have canonical names: $R(G, M)$ is the connected prefix transition graph $P(R)_{M(2)}$ containing a vertex of $M$ (for instance, the first vertex $M(2)$ of $M$ ), where $R$ is the following rewriting system:

$$
R(G)=\left\{p_{X}(s) \stackrel{a}{\longrightarrow} p_{X}(t) \mid \exists H,(X, H) \in G \wedge(s \stackrel{a}{\longrightarrow} t) \in H \wedge a \notin N\right\},
$$

where $p_{X}$ is defined as in Proposition 2.6. We check that $R(G, M)$ is $G^{\omega}(M)$.

We give another definition of $R(G, M)$. Consider a sequence $\left(N_{n}, f_{n}\right)_{n \geqslant 0}$, where $N_{n}$ is a hypergraph with vertices in $V . N^{*}$ and $f_{n}$ is an injection of nonterminal hyperarcs of $N_{n}$ into $N^{*}$. We shall set $R(G, M)=\bigcup_{n}\left[N_{n}\right]$. This sequence is defined as follows:

$$
\begin{aligned}
& N_{0}=\{M\} \quad \text { and } f_{0}(M)=\varepsilon, \\
& N_{n+1}=\left[N_{n}\right] \cup \bigcup\left\{G_{X} \mid X \in N_{n} \wedge X(1) \in N\right\},
\end{aligned}
$$

where for every nonterminal hyperarc $X$ of $N_{n}$ and for the rule $(Y, K)$ of $G$ such that $Y(1)=X(1)$, we have

$$
G_{X}=\left\{f g_{X}\left(s_{1}\right) \ldots g_{X}\left(s_{n}\right) \mid f s_{1} \ldots s_{n} \in K\right\},
$$

where for every vertex $s$ in $K, g_{X}(s)$ is defined by

$$
\begin{aligned}
g_{X}(s)= & s \cdot f_{n}(X) \text { if } s \text { is not a vertex of a nonterminal hyperarc of } K \\
g_{X}(s)= & U(i) \cdot U(1) \cdot f_{n}(X) \text { if } s \text { is the } i \text { th vertex of a nonterminal hyperarc } V \text { of } \\
& K \text { and } U \text { is the left member hyperarc of the rule in } G \text { with the same } \\
& \text { label } U(1)=V(1) \text { as } V \text { (such a } V \text { is unique because } G \text { is separated), }
\end{aligned}
$$

and for every nonterminal hyperarc $Z$ of $G_{X}$,

$$
f_{n+1}\left(Z(1) g_{X}(Z(2)) \ldots g_{X}(Z(|Z|))\right)=Z(1) \cdot f_{n}(X) ;
$$


that is, we push (on the left side) the label of a nonterminal hyperarc before deriving it. So, each vertex in $N_{n}$ is a word giving its place in its pattern (first letter) and the way it was obtained.

Figure 7 gives an example of representative. For every $n \geqslant 0$, we determine the restriction

$$
G_{n}=\{f s t \in R(G, M) \mid d(s, M)<n \vee d(t, M)<n\}
$$

of $R(G, M)$ to the vertices $s$ whose distance $d(s, M)$ at $M$ is at most $n-1$. Then $G_{0}=\emptyset$ and as $G$ is in Greibach form, $G_{n} \subseteq\left[N_{n}\right]$. The grammar $H$, to be constructed satisfying the proposition, must be able to generate from $M$ in $n$ steps of parallel rewritings, a graph having $G_{n}$ as set of terminal arcs. With the exception of $M(1)$, a nonterminal of $H$ will be a couple $(P, Q)$, where $P$ is a finite set of terminal arcs with vertices in $V . N^{*}$, and $Q$ is a subset of vertices of $P$.

Let $n \geqslant 1$. We will determine a set $M_{n}$ of nonterminal hyperarcs allowing the gencration of the graph $R(G, M)-G_{n}$ according to $H$. To this aim, we determine the connected components $D_{1}, \ldots, D_{p}$ of $N_{n+1}-G_{n}$. For $1 \leqslant i \leqslant p$, we consider the set

$$
C_{i}=\left\{f_{s t} t D_{i} \mid f \notin N \wedge\left(s \in V_{G_{n}} \vee t \in V_{G_{n}}\right)\right\}
$$

of terminal arcs of $D_{i}$ having a vertex in $G_{n}$. The hypergraph $M_{n}$ is defined by

$$
\begin{aligned}
M_{n} & =\left\{\left(C_{i} .\left(s_{C_{i}}\right)^{-1},\left(V_{C_{i}} \cap V_{G_{n}}\right) .\left(s_{C_{i}}\right)^{-1}\right) .\left(u_{i, 1}\right) \ldots\left(u_{i, q_{i}}\right) \mid 1 \leqslant i \leqslant p\right. \\
& \left.\wedge\left\{u_{i, 1}, \ldots, u_{i, q_{i}}\right\}=V_{C_{i}} \cap V_{G_{n}} \wedge \forall j, 1 \leqslant j<q_{i}, u_{i, j}<u_{i, j+1}\right\} .
\end{aligned}
$$

The grammar $H$ we look for, is defined as the union of a sequence of grammars $\left(H_{n}\right)_{n \geqslant 1}$. This sequence is inductively constructed as follows:

$$
H_{1}=\left\{\left(M, G_{1} \cup M_{1}\right)\right\}
$$

and

$$
\begin{aligned}
& H_{n+1}=\left\{\left(X .\left(s_{C}\right)^{-1}, C .\left(s_{C}\right)^{-1}\right) \mid X \in M_{n} \wedge X(1) \notin P_{n} \wedge C\right. \text { is the connected } \\
&\text { component of } \left.\left(G_{n+1}-G_{n}\right) \cup M_{n+1} \text { having the vertices of } X\right\},
\end{aligned}
$$

where $P_{n}$ is the set of the nonterminals of $H_{1}, \ldots, H_{n}$.

To prove the finiteness of $H$, it suffices to show that there exists only a finite number of possible nonterminals for $H$. Again, it suffices to find a bound on the distance in $R(G, M)$ of vertices common to $C$ and $G_{n}$, for every $n$ and every connected component $C$ of $R(G, M)-G_{n}$.

Because $G$ is connected, the integer $b=\max \left\{d_{R(G, Y)}(s, t) \mid \exists(Y, K) \in G, s, t \in V_{K}\right\}$ is well-defined. Let us consider a connected component $C$ of $R(G, M)-G_{n}$ for any $n$, and vertices $s$ and $t$ common to $C$ and $G_{n}$. We want to find an upper bound depending on $b$ of the distance $d_{R(G, M)}(s, t)$. Let us take a vertex $u$ of $C$ with minimal length. As $R(G, M)$ is connected, there exists a path of minimal length $d_{R(G, M)}(s, M)$. The grammar $G$ being in Greibach form, this path goes through the "pattern" of $u$, that is 
through a vertex $v$ with the same suffix in $N^{*}$ as $u$, i.e. $v(2) \ldots v(|v|)=u(2) \ldots u(|u|)=s_{C}$. Also $d_{R(G, M)}(u, v) \leqslant d_{R(G, Y)}(u(1), v(1))$ for the rulc $(Y, K)$ of $G$ such that $u(1)$ is a vertex in $K$; hence, $d_{R(G, M)}(u, v) \leqslant b$. Since $s$ is a "border" vertex of $C$ and $G_{n}$, and $u$ is a vertex of $C$, we have $d_{R(G, M)}(s, M) \leqslant d_{R(G, M)}(u, M)$; then

$$
\begin{aligned}
d_{R(G, M)}(s, v)+d_{R(G, M)}(v, M) & =d_{R(G, M)}(s, M) \leqslant d_{R(G, M)}(u, M) \\
& \leqslant d_{R(G, M)}(u, v)+d_{R(G, M)}(v, M) .
\end{aligned}
$$

So, $d_{R(G, M)}(s, v) \leqslant d_{R(G, M)}(u, v) \leqslant b$; hence, $d_{R(G, M)}(u, s) \leqslant d_{R(G, M)}(u, v)+d_{R(G, M)}(v, s) \leqslant 2 b$ and the same holds for $d_{R(G, M)}(u, t)$. Consequently, $d_{R(G, M)}(s, t) \leqslant 4 b$, which is enough to prove the finiteness of $H$. So, there exists an $m$ such that $H_{m+1}=\emptyset$. Then $H=\bigcup\left\{H_{n} \mid 1 \leqslant n \leqslant m\right\}$ is effectively computable because the $H_{n}$ are. By construction $M \in \operatorname{Dom}(H), H$ is uniform, and $R(G, M)$ is isomorphic to $H^{\omega}(M)$; therefore, $H^{\omega}(M)=G^{\omega}(M)$.

The construction in the above proof is illustrated in Fig. 7.

Let us consider the following (nonuniform) grammar $G$ :
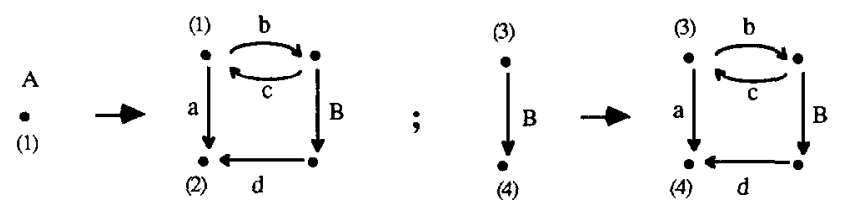

The representative $R(G, A 1)$ of $G^{\omega}(A 1)$ is the following graph:

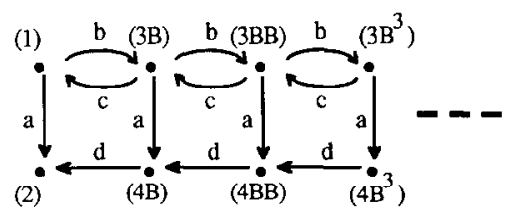

The transformation in the proof of Proposition 2.17 gives the following uniform grammar $H$ :

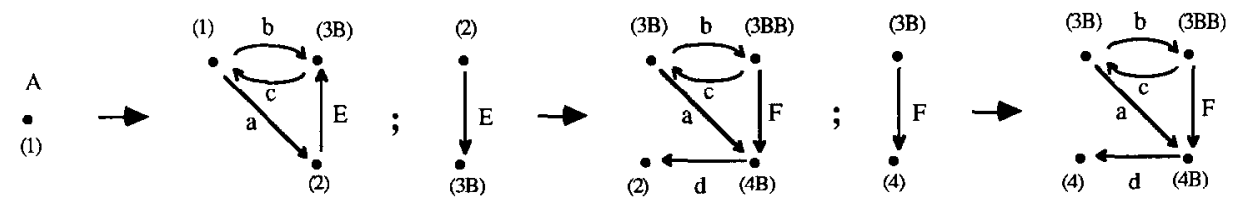

where

$$
\begin{gathered}
E=\left(\left\{3 B \stackrel{b}{\longrightarrow} 3 B B, 3 B B \stackrel{c}{\longrightarrow} 3 B, 3 B \stackrel{{ }^{a}}{\longrightarrow} 4 B, 4 B \stackrel{{ }^{d}}{\longrightarrow} 2\right\},\{2,3 B\}\right) \\
F=(\{3 B \stackrel{b}{\longrightarrow} 3 B B, 3 B B \stackrel{c}{\longrightarrow} 3 B, 3 B \stackrel{a}{\longrightarrow} 4 B, 4 B \stackrel{d}{\longrightarrow} 4\},\{4,3 B\})
\end{gathered}
$$

with the natural order on $\{1,2,3,4\}$ extended lexicographically on $\{1,2,3,4\} . B^{*}$. Hence, $H^{\omega}(A 1)=G^{\omega}(A 1)$.

Fig. 7. Transformation of a grammar into a uniform grammar. 
A noneffective version of Proposition 2.17 has been given by Bauderon [3]. After Propositions 2.17 and 2.7, we can decide that two accessible (connected) prefix transition graphs of word-rewriting systems are isomorphic with respect to some given vertices (i.e. the isomorphism is given on a pair of vertices, say on the roots).

Proposition 2.18. From all triples $\left(R, r, r^{\prime}\right)$ and $\left(S, s, s^{\prime}\right)$ consisting of a rewriting system, $a$ word and a vertex of the accessible (connected) prefix transition graph, we can decide whether there exists an isomorphism from $P(R, r)$ to $P(S, s)\left(\right.$ from $P(R)_{r}$ to $\left.P(S)_{s}\right)$ such that $f\left(r^{\prime}\right)=s^{\prime}$.

Proof. From Proposition 2.7 (Theorem 2.11) and Proposition 2.17, we transform $\left(R, r, r^{\prime}\right)$ into a uniform grammar $G$ and an axiom $a_{0} r^{\prime}$ such that the prefix transition graph of $R$ accessible from $r$ is the graph generated by $G$ from $a_{0} r^{\prime}$, i.e. $P(R, r)=G^{\omega}\left(a_{0} r^{\prime}\right)\left(P(R)_{r}=G^{\omega}\left(a_{0} r^{\prime}\right)\right)$. In the same way, we transform $\left(S, s, s^{\prime}\right)$ into $\left(H, b_{0} s^{\prime}\right)$. Let us denote by $N_{G}\left(N_{H}\right)$ the set of nonterminals in $G(H)$. We will now compare the right members of the uniform grammar rules $G$ and $H$, starting from the right members associated to $a_{0}$ and $b_{0}$ : two such hypergraphs are comparable if there exists an isomorphism identifying their terminal arcs, and associating to every nonterminal hyperarc of the first one, a nonterminal of the other, up to a permutation of vertices. To compare the right members of $G$ and $H$, consider the set $E$ of words $e=(a, b) \pi(1) \ldots \pi(n)$; where $a$ and $b$ are nonterminals of arity $n$, from $G$ and $H$, respectively, and $\pi$ is a permutation of $\{1, \ldots, n\}$. With such a word $e$ of $E$ and given the rules $\left(a s_{1} \ldots s_{n} \rightarrow P\right)$ in $G$ and $\left(b t_{1} \ldots t_{n} \rightarrow Q\right)$ in $H$, we associate the finite set $B_{e}$ of the bijections $h$ of the vertices of $P$ onto the vertices of $Q$, such that the following conditions hold:

$$
\begin{aligned}
h\left(s_{i}\right)=t_{\pi(i)} \quad \text { for } 1 \leqslant i \leqslant n, & \\
c x_{1} \ldots x_{m} \in P \wedge c \notin N_{G} \Leftrightarrow & c h\left(x_{1}\right) \ldots h\left(x_{m}\right) \in Q \wedge c \notin N_{H}, \\
c x_{1} \ldots x_{m} \in P \wedge c \in N_{G} \Rightarrow & \exists d y_{1} \ldots y_{m} \in Q, d \in N_{H} \\
& \wedge\left\{y_{1}, \ldots, y_{m}\right\}=\left\{h\left(x_{1}\right), \ldots, h\left(x_{m}\right)\right\}, \\
d y_{1} \ldots y_{m} \in Q \wedge d \in N_{H} \Rightarrow & \exists c x_{1} \ldots x_{m} \in P, c \in N_{G} \\
& \wedge\left\{y_{1}, \ldots, y_{m}\right\}=\left\{h\left(x_{1}\right), \ldots, h\left(x_{m}\right)\right\}
\end{aligned}
$$

we denote by $E_{e, h}$ the set of such words $(c, d) \sigma(1) \ldots \sigma(m)$ where $c \in N_{G}$ and $h\left(x_{i}\right)=y_{\sigma(i)}$ for $1 \leqslant i \leqslant m$.

Hence, there exists an isomorphism $f$ of $P(R, r)$ onto $P(S, s)$ such that $f\left(r^{\prime}\right)=s^{\prime}$ if and only if there exists a directed unlabelled graph $C$, with vertices in $E$, such that $\left(a_{0}, b_{0}\right) 1$ is a vertex of $C$ and if $e=(a, b) \pi(1) \ldots \pi(n)$ is a vertex of $C$ then there exists a bijection $h$ of $B_{e}$ for which $E_{e, h}$ is the set of targets of arcs in $C$ starting at $e$. Since the set $C$ of such graphs is finite and constructible, we can decide on the isomorphism of $P(R, r)$ and $P(S, s)\left(P(R)_{r}\right.$ and $\left.P(S)_{s}\right)$ associating $r^{\prime}$ with $s^{\prime}$. 
Let us note that Proposition 2.18 is also a consequence of Proposition 2.7 and of Corollary 4.5 of [9].

\section{Acknowledgment}

Let me thank P. Darondeau, R. Monfort and J.-C. Raoult for their help in the drafting of this paper. I also thank some anonymous referees for their numerous remarks.

\section{References}

[1] J.C.M. Baeten, J.A. Bergstra and J.W. Klop, Decidability of bisimulation equivalence for processes generating context-free languages, in: Lecture Notes in Computer Science, Vol. 259 (Springer, Berlin, 1987) 94-111.

[2] M. Bauderon, On systems of equations defining infinite graphs, in: Lecture Notes in Computer Science, Vol. 344 (Springer, Berlin, 1989) 54-73.

[3] M. Bauderon, Infinite hypergraphs: basic properties and systems of equations, Internal Report I-8920, 1989.

[4] L. Boasson and M. Nivat, Centers of context-free languages, Internal Report LITP 84-44.

[5] R. Büchi, Regular canonical systems, Arch. Math. Logik Grundlag. 6 (1964) 91-111.

[6] D. Caucal, Récritures suffixes de mots, Report INRIA 871, 1988.

[7] D. Caucal and R. Monfort, On the transition graphs of automata and grammars, in: $W G$ 90, Lecture Notes in Computer Science, Vol. 484 (Springer, Berlin, 1990) 311-337.

[8] B. Courcelle, The monadic second-order logic of graphs, II: Infinite graphs of bounded width, Math. Systems Theory 21 (1989) 187222.

[9] B. Courcelle, The definability of equational graphs in monadic second order logic, in: ICALP 89 , Lecture Notes in Computer Science, Vol. 372 (Springer, Berlin, 1989) 207-221.

[10] M. Dauchet and S. Tison, The theory of ground rewrite systems is decidable, in: Proc. 5th IEEE Symp. LICS 90, 242-248.

[11] C. Frougny and J. Sakarovitch, Rational relations with bounded delay, in: Proc. 8th STACS 90, Lecture Notes in Computer Science, Vol. 480 (Springer, Berlin, 1990) 50-63.

[12] A. Habel, Hyperedge replacement: grammars and languages, Dissertation, University of Bremen, 1989.

[13] A. Habel and H.J. Kreowski, Some structural aspects of hypergraph languages generated by hyperedge replacement, in: Lecture Notes in Computer Science, Vol. 247 (Springer, Berlin, 1987) 207-219.

[14] D. Muller and P. Schupp, The theory of ends, pushdown automata, and second order logic, Theoret. Comput. Sci. 37 (1985) 51-75.

[15] A. Salomaa, Formal Languages, ACM monograph series (Academic Press, New York, 1973). 